\title{
Giorgio Pighi
}

Università degli Studi di Modena e Reggio Emilia (The University of Modena and Reggio Emilia, Italy) e-mail: giorgio.pighi@unimore.it

ORCID: 0000-0002-1872-749X

DOI: $10.15290 / \mathrm{mhi} .2020 .19 .01 .12$

\section{La tutela penale del minore migrante in Italia}

\begin{abstract}
The criminal protection of the migrant minor in Italy

Providing specific criminal protection for migrant minors requires the balance of two legal aspects related to the specific weaknesses of the children; on the one hand, the condition of the minor is characterized by the inadequate experience and specific risks of victimization; on the other hand, the migrant status is related to the lack of social and family references, economic need and diversity of culture. The protection the two mentioned legal aspects requires the enforcement of both different rules to be combined; the application of existing regulations, with the evaluation of the crime's circumstances, as well as the definition of new criminal figures, who identify some peculiar forms of possible aggression or endangerment, are both useful solutions for the very problematic balance of the two goods. In order to provide effective protection for migrant minors, it is essential to fully implement the principle of "last resort". Criminal protection, in fact, should only intervene in support and completion of priority preventive administrative safeguards; the administrative protection has to intervene before the commission of serious crimes, which risk compromising, even irremediably, the personality of the vulnerable minor. Otherwise, the administrative action would provide only indirect protection, with no effects on weak persons; an efficient intervention aims to preserve the fragility of the migrant child taking into account the objective risks, often very serious, due to the simultaneous of the two conditions (minor age and migration status). Criminal protection alone would not
\end{abstract}


prevent the maturing of trauma, harassment, exploitation, material and educational deprivation. The law of 7 April 2017, n. 47 «Provisions regarding the protection measures for unaccompanied foreign minors» is significant in this regard.

Key words: children, migrant, criminal law, last resort, legal protection, unaccompanied children

\section{Tutela penale, bene giuridico, migranti}

La tutela penale del minore migrante è un'articolazione del più ampio bene giuridico "tutela del minore" e porta a rendere tipiche modalità lesive o di messa in pericolo, riguardanti situazioni o condizioni specificamente nocive per la persona e per la vita sociale, ai danni di questa particolare tipologia di soggetto passivo del reato. Una fattispecie «dedicata», in questi casi, si rende necessaria quando quelle più generali, pur affiancate dagli altri strumenti di tutela, compresi quelli extrapenali, non riescano a raggiungere adeguatamente lo scopo. La tutela delle articolazioni dei beni giuridici, ancorché prossime ai diritti fondamentali della persona umana, al pari di ogni altra tutela, deve rispettare il limite dell'ultima ratio, evitando che la norma penale ecceda la sua funzione e intervenga strumentalmente come «cifra» di invadenti esigenze politiche, più che per l'effettivo bisogno della prevenzione generale propria del reato. Quando la tutela penale seleziona segmenti del bene giuridico di grande impatto, ovvero legati a diritti fondamentali o a situazioni di primaria importanza, oppure quando persegue disvalori particolarmente odiosi, è fondamentale la capacità del legislatore di fornire tutela con esclusivo riguardo al carattere appropriato degli strumenti, non all' impatto sul consenso, che guarda ai contenuti simbolici delle fattispecie.

Un particolare profilo di tutela dei minori migranti ${ }^{1}$, quello rivolto ai minori stranieri non accompagnati ${ }^{2}$, è stato banco di prova molto impegnativo

$1 \quad$ Il d. lgs 18 agosto 2015, n. 142, "Attuazione della direttiva 2013/33/UE recante norme relative all' accoglienza dei richiedenti protezione internazionale, nonché della direttiva 2013/32/UE, recante procedure comuni ai fini del riconoscimento e della revoca dello status di protezione internazionale", all'art. 18 "Disposizioni sui minori», individua compiutamente i nodi problematici della tutela del minore migrante, disponendo che «Nell' applicazione delle misure di accoglienza ... assume carattere di priorità il superiore interesse del minore in modo da assicurare condizioni di vita adeguate alla minore età, con riguardo alla protezione, al benessere ed allo sviluppo anche sociale del minore, conformemente a quanto previsto dall'articolo 3 della Convenzione sui diritti del fanciullo del 20 novembre 1989, ratificata dalla legge 27 maggio 1991, n. 176». La norma, in attuazione dell' art. 31 co. 2 Cost individua conseguentemente gli strumenti, affermando che "per la valutazione dell' interesse superiore del minore occorre procedere all' ascolto del minore, tenendo conto della sua età, del suo grado di maturità e di sviluppo personale, anche al fine di conoscere le 
e affrontato in maniera proficua, nel quale il legislatore si è mostrato attento a non lasciarsi lusingare dalle sirene di questa particolare insidia. Mi riferisco, in particolare, alla 1 . n. $47 / 2017^{3}$ che appresta un sistema preventivo articolato, puntuale e appropriato, costruito esclusivamente con norme extrapenali.

La tutela penale dei minori e quella particolare dei minori migranti sono il frutto d'importanti impegni internazionali assunti dall' Italia sottoscrivendo trattati che fronteggiano il tragico dato d'esperienza del gran numero di situazioni in cui strumenti extrapenali non trovano spazio perché di fatto inesistenti, neppure configurabili ovvero perché concretamente non realizzati. Là dove questi strumenti sono efficacemente attuabili, le norme penali rappresentano invece l'argine imprescindibile per consentire agli interventi proattivi di protezione di produrre i loro effetti positivi.

In alcune situazioni la tutela penale è indispensabile, perché unico rimedio disponibile per contrastare aggressioni intollerabili a chi, assommando le due condizioni, di minore e di migrante, sia doppiamente debole e quindi talmente vulnerabile da rendere inadeguate le due tutele generali - come minore e come migrante - se siano le sole a fronteggiare il gravissimo problema, senza coniugarle fra loro nella tutela complessiva del minore migrante.

La tutela penale dei minori migranti, possiamo dire, è assieme "duplice» e «rafforzata» in quanto raggiunge l'effetto olistico della maggiore efficacia della somma delle due tutele singole, nel perseguire la lesione o la messa in pericolo di una serie di beni giuridici primari (vita, incolumità personale, libertà, autodeterminazione, diritto all'educazione e alla protezione, ecc.) che, ai danni di questi destinatari, assumono spesso connotazioni estreme e odiose o determinano pregiudizi irreversibili nella crescita e nella vita futura.

esperienze pregresse e valutare il rischio che il minore sia vittima di tratta di esseri umani, nonché a verificare la possibilità di ricongiungimento familiare ... purché corrisponda all'interesse superiore del minore». La norma prevede inoltre che i figli minori dei richiedenti e i richiedenti minori siano alloggiati rispettando i vincoli parentali, che siano assicurati i servizi destinati alle esigenze della minore età, comprese quelle ricreative e che gli operatori abbiano qualifiche e formazione adeguate e siano tenuti alla riservatezza.

2 Il d. lgs n. 142/2015, citato alla nota precedente, detta all' art. 19 particolari disposizioni per i minori stranieri non accompagnati, precisando che questi ultimi «sono accolti in strutture governative di prima accoglienza, istituite con decreto del Ministro dell' interno, sentita la Conferenza unificata..., per il tempo strettamente necessario, comunque non superiore a sessanta giorni, alla identificazione e all'eventuale accertamento dell'età, nonché a ricevere, con modalità adeguate alla loro età, ogni informazione sui diritti riconosciuti al minore e sulle modalità di esercizio di tali diritti, compreso quello di chiedere la protezione internazionale». La norma, dopo avere disciplinato le caratteristiche dell' accoglienza e la particolare attenzione da prestare all' assistenza psicologica, precisa che si applicano a questi minori le medesime condizioni previste per i richiedenti asilo dal servizio Sprar.

3 Si veda: B. Triestina, I minori stranieri non accompagnati. Analisi ragionata della L. 7 aprile 2017, n. 47, Piacenza 2017; J. Marzetti, Tutori volontari per minori stranieri non accompagnati, Rimini 2019. 
La tecnica legislativa utilizzata consiste nell' apprestare tutela penale ai minori migranti specializzando o aggravando altre fattispecie, integrando i medesimi reati che tutelano i beni giuridici, della cui offesa il migrante può restare vittima in ogni fase della vita ma che, quando non abbia raggiunto la maggiore età, assumono particolare gravità in ogni caso, ovvero quando la stessa età minore configuri particolari profili di rischio e di disvalore. Quando, invece, la particolare valenza lesiva del fatto, quando commesso nei confronti del minore, non comporti la necessità di rimodellare il fatto tipico, assumerà rilievo nella quantificazione della pena, in termini di gravità del reato, ai sensi dell' art. 133 c.p.

In ogni caso, per dare effettività alla maggiore tutela, va messa in evidenza la particolare cura che il sistema deve avere per presidiare la sicurezza pubblica, attraverso il contrasto efficace del numero oscuro e dell' impunità e rafforzando la capacità di disinnescare le insidie e le situazioni critiche ai danni dei minori migranti, mettendo in campo una forte azione internazionale, per non porre a carico delle politiche sociali e di polizia dei singoli Stati l'intero peso della prevenzione che, così frammentata, non riesce ad avere presa ed incidere sulle caratteristiche criminali dell' intero fenomeNo. Le leggi nazionali, soprattutto nelle emergenze e in presenza di opinioni pubbliche impaurite dai luoghi comuni securitari, sfuggono, spesso per mero opportunismo, alla dimensione transnazionale dei problemi, per non farsi carico della quota-parte del problema, come dimostrano platealmente le difficoltà insorte per accogliere proporzionalmente i profughi e per contrapporsi globalmente alla criminalità transnazionale, al cui interno maturano le situazioni più lesive e più impunite della nostra articolazione del bene giuridico.

\section{La tutela penale del minore}

Per realizzare la tutela penale del minore intervengono fattispecie che specializzano la prevenzione generale della lesione di beni giuridici fondamentali, come la vita, l'integrità fisica, la dignità, la libertà, in attuazione del precetto costituzionale dell' art. $31 \mathrm{co} .2$ che impone di articolare la doverosa protezione dei giovani «favorendo gli istituti necessari a tale scopo».

La Carta fondamentale, per scongiurare che il principio viva nell' ordinamento come semplice esortazione programmatica, dalle ricadute non verificabili, coniuga l'obiettivo con l'obbligo della legge di farsi strumento per realizzarlo con istituti specifici, chiamati a fronteggiare i rischi ai quali le persone giovani e l'infanzia sono esposte in maniera particolare, nelle diverse situazioni di volta in volta disciplinate. La Corte Costituzionale non esita a dichiarare illegittime numerose norme di legge che contraddicono la doverosa tutela differenziata dei minorenni, ravvisando irragionevole ogni trattamento non differenziato delle situazioni, ben diverse, dei minori e degli adulti. Fondamentale fu, come è noto, 
la decisione sull'attenuante della minore età (art. 98 co. 2 c.p.) che deve inderogabilmente produrre effetti concreti sulla pena per qualsiasi condanna, e mai può soccombere a fronte di aggravanti, impedendo, fra l'altro, l'applicazione ai minorenni della pena dell' ergastolo ${ }^{4}$.

La tutela della gioventù e dell' infanzia, sempre doverosa in ogni situazione disciplinata dal diritto, deve essere attuata anche quando carenze, limiti e ritardi complessivi del sistema non le diano concreta attuazione e conseguentemente l'obbligo costituzionale di favorire gli istituti necessari a tale scopo, rafforzato dalla regola internazionalistica del superiore interesse del minore, vive oramai nell'ordinamento come criterio ermeneutico e norma di diretta applicazione.

Rilevante, a questo proposito, è il divario del nostro diritto penale per i minori di sviluppare insufficientemente la loro tutela penale come potenziali vittime, a fronte di una ben più incisiva determinazione per la loro condizione di $\mathrm{rei}^{5}$, pur fondamentale per specializzare, nei loro confronti, le garanzie e il rilievo imprescindibile che deve essere dato all' ancor incompleta esperienza che spesso intreccia il ruolo di reo e di vittima, e all' esigenza di non fare gravare sulla futura vita adulta le conseguenze di tale fragilità.

Anche la tutela penale dei minori vittime di reati deve raggiungere altrettanta adeguatezza di risposte, comparabili con quelle che sono assicurate, grazie anche ai numerosi interventi della Corte Costituzionale, nel trattamento penale differenziato, che consentono di affrontare le peculiarità della delinquenza giovanile senza limitarsi agli accertamenti sulla capacità d'intendere e di volere come presupposto della responsabilità colpevole, per spiegare protezione in funzione del recupero sociale e della prevenzione, rivolti all'intera platea giovanile, con trattamenti puntuali per le diverse tipologie comportamentali.

La legislazione sovranazionale e nazionale, da almeno un trentennio, tende a colmare questo ritardo, rimarcando che la tutela penale dei minori deve apprestare protezione coniugando tale salvaguardia col sistema punitivo differenziato nei confronti del minore che agisce criminosamente, per realizzare un sistema complessivo coerente di protezione quando la condizione giovanile è esposta al rischio di subire lesione o messa in pericolo di beni giuridici significativi ${ }^{6}$.

In alcuni casi il minorenne è soggetto passivo esclusivo di reati che perseguono comportamenti a suo danno che, altrimenti, sarebbero privi di rilievo penale, come nel caso di chi fruisca della prostituzione minorile. In altri casi il reato subito dal minorenne viene punito più severamente, attraverso la specializzazione delle fattispecie o il suo aggravamento. Pensiamo ai fenomeni allarmanti

4 Corte Cost. 28 aprile 1994, n. 168.

5 Si veda: L. Picotti, La tutela penale del minore vittima di reato: profili sistematici e spunti critici, 2018, http://www.aiafrivista.it.

6 F. Nicodemi, Le vittime della tratta di persone nel contesto della procedura di riconoscimento della protezione internazionale: quali misure per un efficace coordinamento tra i sistemi di protezione e di assistenza? "Diritto, immigrazione e Cittadinanza" 2017, no 1, 30 ss. 
dei bambini abusati, maltrattati e violati che prevedono sanzioni più severe, limitazioni alle misure alternative, forme particolari di tutela giudiziaria della dignità, libertà e vulnerabilità delle giovani vittime, assieme alla modulazione delle attività processuali nei confronti di chi compie simili abusi, tese ad assicurare, con particolari norme, l'assunzione della prova e la sua genuinità senza ricadute negative sulle giovani vittime ${ }^{7}$.

La tutela penale dei minori che specializza le differenti offese ai loro danni, incalzata, come detto, dalla normativa sovranazionale, ha portato a nuove incriminazioni o ad articolazioni di quelle generali, per meglio assicurare la tutela della piena dignità umana al giovane, per definizione particolarmente vulnerabile per ragioni legate alla crescita, all' evoluzione e alla maturazione, e quando non sia ancora in grado di difendere autonomamente i propri diritti e, anche per questo, sia maggiormente esposto ad attività criminose, ad aggressioni individuali, a vedere forzato il consenso e l' autodeterminazione . $^{8}$

Alcune fattispecie o disposizioni particolari del nostro codice sono risalenti, mentre altre sono state modellate negli ultimi decenni. Fra le prime rilevano: l'abbandono di persone minori o incapaci (art. 591 c.p.), l'omissione di soccorso (art. 593 c.p.), l'irrilevanza del consenso prestato dal minore degli anni diciotto alla quale consegue la responsabilità per omicidio e non per l'ipotesi più lieve nell'omicidio del consenziente (art. 579 c.p.), l' applicazione delle pene per l'omicidio in caso di istigazione o aiuto al suicidio nei confronti di persona minore degli anni quattordici e una specifica ipotesi aggravata se quest'ultima condotta criminosa sia rivolta a chi abbia meno di diciotto anni (art. 580, co. 2 c.p.).

Quantoallenormedipiù recenteintroduzione, vannoevidenziatel' aggravante speciale dell' omicidio doloso, che comporta la pena dell' ergastolo quando il fatto sia commesso (art. 575 co. 1 n. 5) in occasione del delitto di atti sessuali con minorenne ${ }^{9}$, e le aggressioni alla libertà di movimento, all' autodeterminazione, alla dignità personale, che si concretizzano in modalità di sfruttamento che integrano i delitti di prostituzione minorile (art. 600-bis c.p.), pornografia minorile (artt. 600-ter, 600-quater e 600-quater.1 c.p.), e che perseguono il turismo teso a fruire della prostituzione minorile (art. 600-quinquies c.p.), al fine di combattere lo sfruttamento dei minori a scopo sessuale anche oltre i limiti della territorialità.

7 E. Antonini, La tutela penale dei minori nel Testo Unico sull'immigrazione, [in:] Diritti umani degli immigrati. Tutela della famiglia e dei minori, ed. R. Pisillo Mazzeschi, P. Pustorno, A. Viviani, Napoli 2010.

8 Si veda, in particolare: D. Melossi, M. Giovannetti, I nuovi sciuscià: minori stranieri in Italia, Roma 2002.

9 La modifica è stata inserita dal d.l. 23 febbraio 2009, n. 11, conv. in l. 23 aprile 2009, n. 38. 
Assume importanza significativa, per tutti i reati che vedono il minore rivestire il ruolo di persona offesa dal reato, la disposizione dell' art. 602-quater c.p. ${ }^{10}$ in forza della quale "quando i delitti previsti dalla presente sezione sono commessi in danno di un minore degli anni diciotto, il colpevole non può invocare a propria scusa l'ignoranza dell' età della persona offesa, salvo che si tratti di ignoranza inevitabile». La portata dell'inevitabilità dell'errore sul fatto prevista da questa fattispecie, legata alla lettura data che la Corte Costituzionale ha fatto propria sull' error iuris con la sent. n. 364/1988, è stata puntualizzata dalla giurisprudenza di legittimità ${ }^{11}$, secondo la quale «il fatto tipico scusante previsto dall' art. 602-quater cod. pen. in relazione all' ignoranza inevitabile circa l'età della persona offesa, è configurabile solo se emerga che nessun rimprovero, neppure di semplice leggerezza, possa essere rivolto all' agente, per avere egli fatto tutto il possibile al fine di uniformarsi ai suoi doveri di attenzione, di conoscenza, di informazione e di controllo, attenendosi a uno standard di diligenza direttamente proporzionale alla rilevanza dell'interesse per il libero sviluppo psicofisico dei minori».

La giurisprudenza non si limita a sottolineare che deve sussistere un grado di colpevolezza che renda l' errore rimproverabile, che potrà essere escluso «solo se l'agente, pur avendo diligentemente proceduto ai dovuti accertamenti, sia stato indotto a ritenere, sulla base di elementi univoci, che il minorenne fosse maggiorenne». La prima conseguenza che la giurisprudenza trae è che «non sono sufficienti, al fine di ritenere fondata la causa di non punibilità, elementi quali la presenza nel soggetto di tratti fisici di sviluppo tipici di maggiorenni o rassicurazioni verbali circa l'età, provenienti dal minore o da terzi, nemmeno se contemporaneamente sussistenti». La seconda conseguenza è che il reo «ha l'onere di provare non solo la non conoscenza dell' età della persona offesa, ma anche di aver fatto tutto il possibile al fine di uniformarsi ai suoi doveri di attenzione».

\section{La tutela penale del migrante}

Anche la tutela penale del migrante si sviluppa ordinariamente attraverso una pluralità di reati strumentali rispetto alla lesione di altri beni giuridici, in particolare di quelli che possono più facilmente restare offesi nella particolare condizione di debolezza sociale.

Un primo gruppo di fattispecie rimodella e arricchisce di contenuto taluni reati del codice penale, per adeguarne la struttura alle caratteristiche attuali del fenomeno migratorio, che vede un gran numero di migranti esposti in maniera

10 Introdotta dalla 1.1 ottobre 2012, n. 172.

11 Corte di Cassazione, 24 luglio 2017, n. 36606. 
preoccupante a rischi, un tempo sconosciuti, di violazione dei diritti fondamentali della persona umana e di nuove forme di schiavitù, spesso con modalità subdole e insidiose ${ }^{12}$.

Questa scelta ha portato a riformare profondamente i delitti contro la libertà individuale, previsti dal capo terzo dei delitti contro la persona, per adeguarli alla virulenza, al carattere subdolo e alle odiose peculiarità dei nuovi fenomeni. I delitti contro la personalità individuale della sezione prima (art. 600 e ss. c.p.) sono stati riformulati e arricchiti di fattispecie nuove ${ }^{13}$, con l'aggiunta della sezione I.bis che persegue la propaganda e l'istigazione a delinquere per motivi di discriminazione razziale, etnica e religiosa (art. 604-bis e ter), riconducendo a sistema alcuni reati, fino ad allora contenuti nella legislazione speciale.

Un secondo gruppo di fattispecie, disciplinato dall'art. $12 \mathrm{~d}$. lgs 25 luglio 1998, n. 286, il Testo unico delle disposizioni concernenti la disciplina dell'immigrazione e norme sulla condizione dello straniero, detta disposizioni contro le immigrazioni clandestine attraverso norme incentrate sulla tutela dei confini nazionali, che articolano il bene giuridico mettendo in valore anche le differenti condizioni di fragilità dello straniero che viene fatto entrare illegalmente in Italia.

La fattispecie base del co. 1 punisce con la reclusione da uno a cinque anni e con la multa di 15.000 euro per ogni persona chiunque compie una serie di attività dirette a favorire l'ingresso degli stranieri nel territorio dello Stato «in violazione delle disposizioni del presente testo unico». La disposizione articola la tutela del bene comminando pene più elevate (reclusione da cinque a quindici anni e multa di euro 15.000 per ogni persona) in relazione al numero dei concorrenti nel reato, all'esposizione a pericolo per la vita, alla sottoposizione a trattamenti inumani o degradanti, al numero delle persone fatte entrare illegalmente, all' utilizzo di mezzi di trasporto internazionali, al ricorso a documenti contraffatti, alla disponibilità di armi ed esplosivi.

Il co. 3-ter aggrava da un terzo alla metà la pena della reclusione e porta la multa a euro 25.000 per ogni persona, qualora ricorrano alcune condizioni sog-

12 E. Antonini, op cit.

13 L'attuale disciplina è frutto di una lenta e tormentata evoluzione. La l. 3 agosto 1998, n. 269 introdusse i reati di prostituzione minorile (art. 600-bis c.p.), pornografia minorile (art. 600-ter c.p.), detenzione di materiale pornografico realizzato utilizzando minori degli anni diciotto (art. 600-quater c.p.), iniziative turistiche volte allo sfruttamento della prostituzione minorile (art. 600-quinquies. La 1.1 ottobre 2012, n. 172 ha modificato i richiamati artt. 600-bis e 600-ter. La 1. 11 agosto 2003, n. 228 ha riformulato le fattispecie dei delitti di schiavitù, servitù e tratta ( artt. 600, 601 e 602 c.p.) che sono state modificate dal d. lgs 4 marzo 2014 , n. 24. La l. 15 luglio 2009, n. 94 ha introdotto il nuovo delitto di «impiego di minori nell' accattonaggio» all' art. 600-octies c.p., successivamente modificato. Il reato di pornografia virtuale (art. 600-quater.1) è stato introdotto dalla 16 febbraio 2006, n. 38. Le circostanze aggravanti per i reati di cui agli artt. 600, 601 co. 1 e 2 e 602 previsti dall' art. 602-ter sono stati inseriti dalla 1. 2 luglio 2010, n. 108. 
gettive degli stranieri fatti entrare illegalmente, che danno vita al dolo specifico rappresentato dal fine di reclutamento di persone da destinare alla prostituzione o allo sfruttamento della prostituzione e rendono tipica la particolare condizione futura dei soggetti passivi quando si tratti di minori da impiegare in attività illecite al fine di favorirne lo sfruttamento. Va rilevato che la norma dispone che queste circostanze aggravanti non possano soccombere o essere ritenute equivalenti - e quindi perdere la traduzione del disvalore che esprimono - in presenza di attenuanti (commi 3-quater e 3-quinquies).

L'espressione «da impiegare», pur non particolarmente precisa, non consiste in un dolo specifico che rende tipico l'agire finalistico del soggetto attivo del favoreggiamento dell'immigrazione clandestina, ma nel movente della migrazione che portò il minore, o altri per lui, tra i quali può rientrare lo stesso favoreggiatore, a perseguire l'ingresso nel territorio dello Stato in violazione delle disposizioni del t.u., in vista dell' impiego illecito indicato. Trattandosi di circostanza aggravante, il reo ne risponderà se ne era a conoscenza o se tale movente era conoscibile, ma fu ignorato per colpa o ritenuto inesistente per errore determinato da colpa (art. 59 co. 2 c.p.).

La condanna per favoreggiamento dell' immigrazione clandestina, nell' ipotesi semplice e in quelle aggravate, fra le quali rientra quella dei soggetti passivi minorenni da impiegare in attività illecite al fine di favorirne lo sfruttamento, comporta il regime carcerario dei reati di maggiore gravità e preclude, con la sola eccezione di chi collabora con la giustizia, l'assegnazione al lavoro all'esterno, i permessi premio, e le misure alternative alla detenzione, in conseguenza di specifica norma contenuta nell' art. 12 co. 3-sexies del t.u. sull'immigrazione, che modifica l'art. 4-bis dell'Ordinamento penitenziario (1. n. 354/1975).

\section{La tutela penale del minore migrante}

Anche i nuovi delitti previsti nel codice penale, profondamente rimaneggiati sotto la spinta della trasformazione fenomenologica dei crimini legati ai fenomeni migratori ${ }^{14}$, al pari di quanto si è verificato per la richiamata figura del favoreggiamento dell'immigrazione clandestina, contengono norme poste a tutela penale del minore migrante. Due dei delitti in questione, in particolare, sono costruiti sulla condizione di fragilità alla quale deve conseguire l'assommarsi delle due tutele $\mathrm{e}^{15}$.

14 Si veda: K. Summerer, I delitti di schiavitù e tratta delle persone, [in:] Trattato di diritto penale, Vol. VIII, ed. A. Cadoppi, S. Canestrari, A. Manna, M. Papa, Torino 2010, pp. 213 ss.

15 M. Gambini, I diritti del minore vittima di tratta e gli strumenti di tutela della persona, "Costituzionalismo.it" 2014. 
Il delitto di tratta di persone, previsto dall'art. 601 c.p. ${ }^{16}$ punisce alcune condotte tipiche (reclutare, introdurre nel territorio dello Stato, ecc.) che integrano condizioni lesive di soggezione continuativa, individuate nella sostanziale schiavitù descritta dall' art. 600 c.p. ${ }^{17}$, o nel trovarsi prigionieri di tale soggezione perché rimasti vittime di inganno, violenza, minaccia e di altre modalità di prevaricazione specificamente indicate. Va sottolineato che le pronunce giurisprudenziali hanno rilevato situazioni, nello svolgersi dei processi, che fanno emergere un quadro di immensa gravità ${ }^{18}$.

Il secondo comma dell'art. 601 c.p. dispone che «alla stessa pena soggiace chiunque, anche al di fuori delle modalità di cui al primo comma, realizza le condotte ivi previste nei confronti di persona minore di età». Il fatto tipico differenzia, ampliandola, la tutela penale del minore e prevede che integri reato la semplice esistenza della soggezione continuativa ai sensi dell'art. 600 c.p. o la mera realizzazione delle condotte tipiche (reclutare, introdurre nel territorio dello Stato, ecc.) ai danni di un minorenne, anche senza inganno, violenza, ecc. ${ }^{19}$, senza che debbano ricorrere gli altri elementi descritti nella condotta tipica.

L'art. 602-ter articola disvalori particolari in alcune circostanze aggravanti speciali per i reati di cui agli artt. 600, 601 co. 1 e 2, e 602 c.p. che comportano aumento della pena da un terzo alla metà, assoggettandole, anche in questo caso, alla particolare disciplina che le esclude dal giudizio di bilanciamento (art. 69 c.p.) in termini di equivalenza o soccombenza rispetto ad attenuanti che, conseguentemente, opereranno una più contenuta diminuzione, intervenendo sulla pena già aumentata in modo consistente per effetto delle aggravanti ${ }^{20}$.

16 Si veda: A. G. Cannavale, C. Lazzari, Tratta di persone (voce), [in:] Dig. Disc. Pen. terzo aggiornamento, Torino 2005, p. 1721 ss.

17 Secondo la giurisprudenza, «la condizione di integrale asservimento, necessaria per l'integrazione del delitto di riduzione in schiavitù, non è incompatibile con una certa libertà di movimento, che potrebbe, anzi, essere funzionale alla sua realizzazione - come nel caso in esame quanto all' attività di accattonaggio - ed è concetto diverso dalla totale privazione della libertà personale, che, invece, ad esso non risulta essenziale, essendo elemento tipico del delitto di sequestro di persona di cui all' articolo 605». La Corte, al riguardo, afferma la «non decisività, ai fini dell'integrazione del delitto, di una completa privazione della libertà personale, al ricorrere di specifiche condotte violente da parte dell' imputato, alla continuativa pratica di accattonaggio cui era costretta la persona offesa, alla evidente condizione di inferiorità fisica e psichica, essendo la stessa all' epoca dei fatti tredicenne e per di più incinta». Così: Cassazione, 7 giugno 2016 n. 23590.

18 C. Bianchelli, Il (dis)crimine della tratta. Un'indagine etnografica dei processi penali per riduzione in schiavitù e tratta di esseri umani, "Problemi del socialismo" 2016, LV, p. 77 ss.

19 La portata della norma, precisata in giurisprudenza, comporta che «la nuova formulazione dell' articolo 601 c.p., la cui ratio risiede nell' aggravamento del regime relativo alla tratta dei minori, non abbia provocato alcun assorbimento dell' aggravante di cui all' articolo 602 ter c.p. nella fattispecie semplice ma abbia soltanto chiarito la condotta di cui al primo comma, allorquando oggetto della tratta siano soggetti minori, nel senso che la condotta della tratta è configurabile anche in assenza delle modalità esplicitate nel primo comma nell'ipotesi di minori quali soggetti passivi del contestato delitto». Così: Cassazione, 1 ottobre 2015, n.39797.

20 La Corte di Cassazione, con la sentenza citata alla nota precedente, afferma che «il delitto in questione si ravvisa anche se una persona libera sia condotta con inganno in Italia, al fine di porla nel 
L'art. 602-ter co. 1 c.p., aggravante speciale ad effetto speciale, aumenta la pena da un terzo alla metà "se la persona offesa è minore degli anni diciotto", per i reati di riduzione o mantenimento in schiavitù (art. 600 c.p.), di tratta di persone (art. 601 c.p.) e di acquisto e alienazione di schiavi (art. 602 c.p.). Il co. 4 prevede eguale aumento di pena per i reati di induzione, favoreggiamento, sfruttamento della prostituzione minorile e assimilati (art. 600-bis co. 1 e 2 c.p.), pornografia minorile (art. 600-ter co 1 c.p.) e iniziative turistiche volte allo sfruttamento della prostituzione minorile (art. 600-quinquies c.p.), «se il fatto è commesso approfittando della situazione di necessità del minore». Il co. 5 prevede che, negli stessi casi indicati dai co. 1 e 4 e in alcune altre ipotesi, la pena sia aumentata dalla metà ai due terzi «se il fatto è commesso in danno di un minore degli anni sedici». Il co. 6 indica i casi in cui la pena è aggravata - e quindi nel caso di minorenni sarà ulteriormente aggravata - quando il fatto è commesso ai danni di persona legata da vincoli familiari o di convivenza.

La disciplina complessiva della tutela penale del minore è sorretta da speciali norme processuali funzionali alla sua effettiva realizzazione. Mi riferisco, in particolare, alla partecipazione del minore al procedimento penale prevista nella Convenzione Onu sui diritti del fanciullo del 1989, con particolare riguardo al diritto del minore di essere ascoltato in ogni procedura giudiziaria, ribadito nella Carta dei diritti fondamentali dell' Unione europea, allegata al Trattato di Lisbona entrato in vigore l' 1 dicembre 2009, nella quale si afferma che «in tutti gli atti relativi ai bambini, siano essi compiuti da autorità pubbliche o da istituzioni private, 1 'interesse superiore del bambino deve essere considerato preminente» ${ }^{21}$.

Il Consiglio dell' Unione europea con una decisione quadro che rimarca con puntualità la tutela della vittima nel procedimento penale ${ }^{22}$ prima, durante e dopo il procedimento penale, mette in luce profili che, pur dettati in via generale per tutte le tipologie di vittime, determinano ricadute molto forti sulla tutela penale dei minori migranti, in quanto vittime particolarmente vulnerabili, che devono beneficiare di trattamenti specifici, che rispondano in modo ottimale

nostro territorio in condizione analoga alla schiavitù; il reato di tratta può essere, infatti, commesso anche con induzione mediante inganno in alternativa alla costrizione con violenza o minaccia. A tal proposito la novella di cui al d. lgs 4 marzo 2014, n. 24 lungi dal modificare sostanzialmente la disciplina della fattispecie delittuosa di cui all' articolo 601 c.p., ha semplicemente precisato in dettaglio le modalità attraverso le quali si realizza la tratta di esseri umani. Inoltre, ai fini della consumazione del reato di tratta di persone, con riguardo alla seconda delle ipotesi previste dall' art. 601, co. primo c. p., non è neppure necessario che venga consumato anche il reato di riduzione in schiavitù, quale previsto dalla richiamata norma, atteso che con tale richiamo si è inteso soltanto, da parte del legislatore, stabilire la necessità del dolo specifico da cui la condotta dell' agente dev' essere accompagnata, nulla rilevando, quindi, che la finalità da lui perseguita non si realizzi, ovvero si realizzi ad opera di soggetto diverso, non necessariamente concorrente con il primo».

21 Si veda: Manuale sul diritto europeo in materia di asilo, frontiere e immigrazione, Agenzia dell' Unione europea per i diritti fondamentali 2014, https://fra.europa.eu.

22 Trattasi della Decisione quadro del Consiglio dell' Unione europea del 15 marzo 2001, relativa alla posizione della vittima nel procedimento penale, n. 2001/220/GAI, https://eur-lex.europa.eu. 
alle esigenze legate alla loro situazione. La decisione, infatti, afferma che «ciascuno Stato membro assicura che le vittime particolarmente vulnerabili beneficino di un trattamento specifico che risponda in modo ottimale alla loro situazione». L'Unione europea, in generale, identifica come vittima la "persona fisica che ha subito un pregiudizio, anche fisico o mentale, sofferenze psichiche, danni materiali causati direttamente da atti o omissioni che costituiscono una violazione del diritto penale di uno Stato membro» e ne fa quindi questione di tutela effettiva, non di mera presenza nell' ordinamento di norme necessariamente specifiche sulle diverse situazioni.

\section{I minori stranieri non accompagnati: una condizione che esige speciale tutela}

Nel quadro della tutela penale dei minori, l'articolazione rappresentata dai minori migranti ha assunto un ruolo di primo piano in conseguenza della profonda trasformazione dei fenomeni migratori e del moltiplicarsi di forme un tempo sconosciute di sfruttamento e di lesione dei diritti fondamentali e della dignità umana, allarmanti per gravità e intensità e neppure pensabili in società che hanno debellato, da secoli, le forme più bestiali di soggiogamento.

Il nodo della mancata protezione dei minori migranti, ai quali la legge ha cercato di porre argine, consiste nell' individuare, innanzitutto, adeguate misure proattive per contrastare ben definiti rischi ai quali essi sono esposti fronteggiando molteplici criticità col dovuto rigore, superando le situazioni di debolezza del sistema nel farsene carico e persino comportamenti conniventi da parte degli adulti che dovrebbero accudirli.

Emergono contorni di fenomeni nuovi, legati in maniera particolare ai flussi irregolari dei migranti, tra i quali spicca, per importanza, quello dei minori stranieri non accompagnati, categoria definita normativamente di destinatari della protezione, che delinea una situazione aperta a molteplici rischi, rispetto ai quali il legislatore si è interrogato per individuare le risposte più efficaci, non accontentandosi di dare visibilità ai nodi problematici attraverso l'ennesimo ritocco delle fattispecie penali, spesso autoassolutorio rispetto a ritardi e carenze imperdonabili.

Appare condivisibile, per alcune evidenti ragioni, la scelta di considerare sufficienti e adeguate le fattispecie penali già esistenti, peraltro rimodellate di recente, per concentrarsi, invece, su innovazioni di carattere organizzativo e sociale, per realizzare una effettiva protezione scandita puntualmente da procedimenti verificabili.

Per prevenire gravi fatti criminosi ai danni dei minori stranieri non accompagnati, accanto alla conoscenza approfondita delle drammatiche peculiarità dei fenomeni, va affrontato e risolto il delicato problema di una particolare condi- 
zione giuridica, che i legislatori hanno teso a definire, in rapporto a un fenomeno in precedenza sconosciuto, affrontando significative difficoltà. Basti pensare che il nostro ordinamento prendeva in considerazione la sola mancanza delle figure dei genitori e di chi potesse esercitare il ruolo parentale al loro venir meno, problema ben diverso, al quale, secondo una consolidata tradizione giuridica, si dà risposta con la responsabilizzazione della collettività e in particolare del Comune di residenza ${ }^{23}$.

La disciplina richiamata affronta un problema irrimediabilmente altro rispetto alla condizione dei minori stranieri non accompagnati, persino sotto il fondamentale profilo di quale autorità debba occuparsene.

Oggi, finalmente, i destinatari della protezione, secondo la definizione che ne dà la 1. n. 47/2017 (c.d. "Legge Zampa"), sono le persone non ancora diciottenni, prive di cittadinanza italiana o dell' Ue, che si trovano nel territorio nazionale prive di assistenza e di rappresentanza dei genitori o di altri adulti legalmente responsabili.

Ovviamente la competenza comunale, espressione del territorio in cui il problema va gestito ha caratteristiche completamente diverse, trattandosi di bambini e ragazzi senza alcun legame preesistente, il cui numero e le cui condizioni individuali non sono né programmabili né prevedibili, che coinvolge ragazzi e bambini spesso reduci da esperienze subite, anche tragiche, che testimoniano un bagaglio di vita pesante alle spalle, nel quale ricorrono violenze, torture, schiavitù, privazioni. Queste situazioni traumatiche si assommano ad esperienze di viaggi durati mesi se non anni, senza adulti di riferimento, per raggiungere un futuro possibile in Europa.

Anche sul piano fenomenologico le caratteristiche di questa tipologia di minori sono peculiari ${ }^{24}$. Grande rilievo hanno, infatti, gli ostacoli all'integrazione

23 Il già citato d. lgs 18 agosto 2015, n. 142, "Attuazione della direttiva 2013/33/UE recante norme relative all' accoglienza dei richiedenti protezione internazionale, nonché della direttiva 2013/32/UE, recante procedure comuni ai fini del riconoscimento e della revoca dello status di protezione internazionale", (c.d. decreto accoglienza), dettò le prime disposizioni specifiche in tema di accoglienza dei minori non accompagnati, per superare l'incongrua applicazione delle norme per i minori in stato di abbandono, operando la distinzione tra prima accoglienza (per le esigenze di soccorso e di protezione immediata, in accordo col Comune nel cui territorio è situata la struttura, e gestite dal Ministero dell'interno anche in convenzione con i comuni) e di seconda accoglienza all'interno del sistema Sprar, con divieto di inserimento in Centri di permanenza per i rimpatri e in altri centri di prima accoglienza. Significativa, al riguardo, l'individuazione dei soggetti vulnerabili (art. 17): «Le misure di accoglienza previste dal presente decreto tengono conto della specifica situazione delle persone vulnerabili, quali i minori, i minori non accompagnati, i disabili, gli anziani, le donne in stato di gravidanza, i genitori singoli con figli minori, le vittime della tratta di esseri umani, le persone affette da gravi malattie o da disturbi mentali, le persone per le quali è stato accertato che hanno subito torture, stupri o altre forme gravi di violenza psicologica, fisica o sessuale o legata all' orientamento sessuale $\mathrm{o}$ all' identità di genere, le vittime di mutilazioni genitali».

24 V. Cianciolo, La tutela del minore straniero non accompagnato, Maggioli 2019. 
trattandosi di irregolari (sans papier) e, nello stesso tempo, non accompagnati e quindi soli, che diventeranno adulti in un Paese che non conoscono, chiamati ad affrontare le difficoltà di integrazione, che incideranno sul loro futuro, prime fra tutte il doveroso apprendimento della lingua e l' inserimento scolastico e lavorativo $^{25}$, in un quadro di opportunità d'integrazione difficili da ottimizzare, poiché gli affidi familiari, certamente lo strumento più idoneo, riescono solo in parte a farsi carico di loro ${ }^{26}$.

Il problema è reso ancora più grave dal numero esorbitante di fughe di questi minori dai luoghi di accoglienza, derivanti dai singoli obiettivi di vita futura, rispetto ai quali il "passaggio» in Italia mira a sfuggire al «controproducente», per così dire, luogo di prima identificazione, disciplinato dagli accordi internazionali, rispetto al più agevole raggiungimento della «meta» che culminerà, passando da soli i confini, nel ricongiungimento con familiari o amici, che comporta la mancanza, per periodi anche lunghi, di alloggio, protezione e assistenza, a fronte di pesanti rischi di isolamento, abusi e lesione dei diritti fondamentali.

Un quadro umano e sociale così problematico ha portato il legislatore a tutelare questi minorenni, assecondando le particolari esigenze di salvaguardia, senza ricorrere agli specifici meccanismi generalpreventivi del sistema penale, ritenendo adeguati quelli già previsti per assolvere al compito di contrastare chi favorisce tanta disumanità. Ha pertanto valorizzato gli strumenti proattivi, articolandoli secondo una pluralità di azioni atte a soddisfare l'esigenza di tenerli lontani dal complesso di situazioni che determinano elevata esposizione a fattori criminogeni e significativa vittimizzazione ${ }^{27}$.

25 A. Di Nuzzo, Minori migranti. Nuove identità transculturali, Carocci 2020.

26 A. Anzaldi e T. Guarnier (ed.), Viaggio nel mondo degli stranieri non accompagnati: un'analisi giuridico-fattuale, Vol. II, In bilico fra il diritto al lavoro e lo sfruttamento, http://www.fondazionebasso. it.

27 I programmi di protezione dei minori stranieri non accompagnati furono avviati con la sperimentazione di un sistema in rete, finanziando i comuni per la presa in carico, giungendo a risultati rilevanti nello standard di accoglienza e nell' utilizzo efficiente delle risorse pubbliche. Va ricordato che la materia dell'immigrazione è di competenza statale nel quadro del coordinamento con le Regioni (art. 117 e 118 co. 3 Cost.); in altre parole, la Costituzione prevede una riserva di legge statale nel coordinamento tra lo Stato e le Regioni. In materia trova applicazione il TUEL n. 267/2000 che prevede, qualora il Comuna assolva compiti nazionali, l'obbligo dello Stato di assicurare la copertura con adeguati trasferimenti. Il Fondo nazionale per i minori stranieri non accompagnati fu istituito per superare la forte disomogeneità di interventi che erano attuati nei diversi Comuni e Regioni a favore di questa particolare categoria di migranti. Già l'art. 37-bis della 1. 184/1983 aveva affermato la competenza statale, stabilendo che «al minore straniero che si trova nello Stato in situazione di abbandono si applica la legge italiana in materia di adozione, di affidamento e di provvedimenti necessari in caso di urgenza», e le disposizioni furono rafforzate dalla Convenzione ONU del 1991sui diritti dell' infanzia, che fu ratificata con 1.27 maggio $1991 \mathrm{n}$. 176, nella quale si afferma la specificità della condizione minorile e viene proclamato il principio che l'interesse superiore del minore va considerato preminente per ogni atto che lo concerne. Il divieto di espulsione del minore previsto dall' art. 19 del Tu n. 286/98 sull' immigrazione rappresenta una modalità di attuazione molto avanzata di questo principio, rispetto al quadro internazionale. Il richiamato coordinamento fra Stato ed Enti locali comporta la creazione di un sistema nazionale, frutto dell'intesa fra tutti gli attori 
Il perseguimento reattivo dei reati ai danni dei minori stranieri non accompagnati è soddisfatto delle nuove fattispecie incriminatrici a tutela dei minori migranti, che appaiono spesso particolarmente calzanti. Non vi è necessità di modulare in modo specifico la vittimizzazione dei minori stranieri non accompagnati, quando siano accertati i delitti, approfonditi nel terzo paragrafo, di riduzione in schiavitù, prostituzione minorile, tratta di persone fattispecie che già apprestano tutela ai minori, con differenti modalità. Se la condizione di non accompagnati, fra l' altro, dovesse incidere sul disvalore del fatto, il giudice ne terrà conto nella quantificazione della pena.

La tutela rafforzata del minore migrante che considera come ultima ratio la risorsa penale, con scelta ragionevole, è stata ritenuta sufficiente per soddisfare la situazione particolare, le cui peculiarità richiedono soprattutto interventi extrapenali per far fronte ad un fenomeno che si sviluppa in numerose direzioni, le cui criticità, impongono agli stati di liberarsi da una situazione di costante emergenza ${ }^{28}$, e che possono essere così riassunte:

- dare effettiva applicazione al divieto assoluto di respingimento del minore non accompagnato alla frontiera e alla possibilità di disporre l'espulsione dello stesso solo a condizione, comunque, che il provvedimento non comporti rischi di danni gravi per il destinatario;

- realizzare strutture di prima accoglienza esclusivamente per tali minori, prevedendo un periodo molto breve di permanenza, al quale segue una collocazione più stabile e duratura;

- ricostruire, nel pieno riconoscimento del diritto all'identità, la storia personale e familiare, evidenziando gli elementi utili a predisporre la soluzione di lungo periodo, più rispondente al superiore interesse del minore $^{29}$;

- tutelare l'unità familiare attraverso indagini svolte nell'esclusivo interesse del minore ${ }^{30}$, ad esito delle quali l'affidamento potrà avvenire esclusivamente se saranno individuati familiari idonei a prendersene cura,

istituzionali coinvolti, non solo per garantire interventi uniformi e di qualità su tutto il territorio nazionale, ma anche per favorire, grazie alla collaborazione con le Regioni, una più equa ripartizione dei destinatari. Il sistema, infatti, deve rispondere a un disegno omogeneo che coinvolga le politiche scolastiche, di formazione, lavorative, abitative e disostegno sociale, capace di valorizzare le esperienze e le competenze già acquisite nei territori, per dare ad esse continuità, favorendo azioni sinergiche tra tutte le istituzioni coinvolte.

28 Si veda: Piccoli schiavi invisibili 2019. Rapporto sui minori vittime di tratta e grave sfruttamento, IX edizione, Save the Children, https://s3.savethechildren.it; e, quanto all'incidenza dell'epidemia da Covid-19 sulla tratta dei minori, cfr.: Idem, Piccoli schiavi invisibili 2020.L'impatto del COVID-19 sulla tratta e lo sfruttamento: dalle strade all'online, ivi.

29 E. Lamarque, Prima i bambini. Il principio dei best interests of the child nella prospettiva costituzionale, Milano 2016.

30 R. Borello, Il diritto all'unità familiare nel diritto dell'immigrazione: riflessioni generali di diritto costituzionale interno e comparato, [in:] Diritti umani degli immigrati. Tutela della famiglia e dei minori, (ed.) R. Pisillo Mazzeschi, P. Pustorno, A. Viviani, Napoli 2010. 
disponendo eccezionalmente, o in assenza di affidatari disponibili, il collocamento in comunità e garantendo il rimpatrio assistito e volontario $^{31}$;

- dare piena attuazione al diritto a salute, istruzione e apprendistato, col rilascio di titoli conclusivi degli studi intrapresi anche se sia sopraggiunta la maggiore età (32).

\section{La legge 7 aprile 2017, n. 47 «Disposizioni in materia di misure di protezione dei minori stranieri non accompagnati»}

La legge n. 47/2017 ha seguito la strada di rafforzare la tutela dei diritti dei minori stranieri non accompagnati uniformandosi ai suddetti obiettivi, da tempo indicati dagli organismi di protezione nazionale e internazionale ${ }^{33}$, rafforzata nell' efficacia generalpreventiva delle norme penali già esistenti, senza ricorrere a specifica disciplina penalistica, ritenuta superflua in forza del già intervenuto aggiornamento delle fattispecie incriminatrici ${ }^{34}$.

In un quadro di tutela penale opportunamente ridisegnato in funzione dei gravi rischi ai quali i fenomeni migratori espongono i minori, va apprezzata la cura dimostrata nella scansione degli strumenti di prevenzione generale proattiva, tesi a realizzare un sistema protettivo non emergenziale - in vero molto ambizioso - che parte dall' affermazione di principio (art. 1) secondo la quale «i minori stranieri non accompagnati sono titolari dei diritti in materia di protezione dei minori a parità di trattamento con i minori di cittadinanza italiana o dell'Unione europea».

Il Parlamento ha approvato la legge in esame a seguito di indagine conoscitiva che ha coinvolto organizzazioni, istituzioni e qualificati esperti del settore, sancendo importanti principi, quali il divieto assoluto di respingimento alla frontiera dei minori stranieri non accompagnati e ha modificato la disciplina del divieto di espulsione aggiungendo all' unica ipotesi in cui è consentita (motivi di ordine pubblico e sicurezza dello Stato), la condizione che non ricorra rischio di danni gravi per il minore.

La procedura d'identificazione, rispetto alla quale appaiono elevati i rischi di invasività lesiva della dignità personale, deve ora svolgersi con unica moda-

31 L. Miazzi, Il rimpatrio assistito del minore straniero: ancora un caso di diritto speciale?, "Diritto Immigrazione e Cittadinanza" 2000, no 2.

32 R. Bertozzi, Le politiche sociali per i minori stranieri non accompagnati, Milano 2005.

33 R. Bichi (ed.), Separated children. I minori stranieri non accompagnati, Milano 2008; G. Tarzia, Il minore straniero non accompagnato: quale tutela nel sistema legislativo italiano?, "Minori Giustizia" 2008, no 3.

34 Si vedano: La tutela dei minori stranieri non accompagnati. Manuale operativo, Ministero dell' Interno - Anci, www.siproimi.it; Il sistema normativo a tutela dei minori stranieri non accompagnati, Unhcr, Unicef e altri, in www.unhcr.it; La tutela dei minori stranieri non accompagnati. Manuale giuridico per l'operatore, Asgi, www.asgi.it. 
lità, accertando l'effettiva minore età in modo attento a salvaguardare i diritti fondamentali ${ }^{35}$. Molto incisiva è la disciplina dell' accoglienza, che rafforza con modalità appropriate, il ruolo dei Comuni in funzione delle già rimarcate esigenze, riconoscendo ai minori la fruizione dei servizi territoriali e disponendo che, al compimento della maggiore età, i richiedenti asilo rimangano nei centri di seconda accoglienza (Sprar) fino alla definizione della domanda di protezione internazionale.

Viene resa più celere l'indagine familiare dando preferenza, per legge, agli affidamenti familiari rispetto al collocamento in comunità, attraverso disposizioni specifiche ${ }^{36}$. La legge prevede inoltre la possibilità di rilasciare, due differenti titoli di legittimazione a rimanere nello Stato: il permesso di soggiorno per minore età ovvero quello per motivi familiari, disponendo che quest' ultimo sia ottenibile quando ricorra la presenza della tutela legale in capo a un cittadino italiano ovvero a un cittadino straniero regolarmente soggiornante e convivente con il tutore, ovvero quando il minore sia a lui affidato, oppure sia affidato di fatto a parente entro il quarto grado. L' originaria collocazione nel sistema Sprar (servizio protezione richiedenti asilo e rifugiati, smantellato dal D.l. n. 113/2018 e sul quale il legislatore pare orientato a tornare sui propri passi), ha assunto la nuova denominazione Siproimi (Sistema di protezione per titolari di protezione internazionale e minori non accompagnati).

Viene inoltre prevista l' istituzione del Sistema informativo nazionale dei minori stranieri non accompagnati, presso il Ministero del lavoro e delle politiche sociali, nel quale confluiscono le singole cartelle sociali, compilate nella fase di prima accoglienza. Dai dati raccolti da tale sistema risulta che, nel 2019, sono stati censiti 6.054 minori stranieri non accompagnati, dei quali 5.737 maschi e 317 femmine ${ }^{37}$.

Viene estesa l'assistenza sanitaria con l'iscrizione al Servizio sanitario nazionale, prima prevista solamente in presenza di permesso di soggiorno già rilasciato. Tale assistenza è comunque garantita sin dal momento del rintraccio sul territorio nazionale e sono incentivate misure delle istituzioni scolastiche e formative tese a garantire l'assolvimento dell'obbligo scolastico e formativo. Sul piano dei diritti personali, vengono rafforzate le garanzie sul piano della tutela della persona e della tutela legale, assicurando, nei diversi procedimenti, assistenza affettiva e psicologica, i diritti di: essere informato, nominare un legale

35 Si veda: Norme sull' accertamento dell' età dei minori stranieri non accompagnati, ASGI, (23.01.2017), https://www.asgi.it/asilo-e-protezione-internazionale/minori_accertamento_eta_regolamento

36 Si veda la scheda illustrativa e riepilogativa: , Minori stranieri non accompagnati, Camera dei deputati, XVIII legislatura, https://temi.camera.it/leg18/temi/tl18_minori_stranieri_non_accompagnati. html

37 Si veda: Report mensile minori stranieri non accompagnati (msna) in Italia, Ministero del lavoro e delle politiche sociali, Direzione Generale dell' Immigrazione e delle Politiche di Integrazione, Dati al 31 dicembre 2019, in www.lavoro.gov.it. 
di fiducia e fruire di gratuito patrocinio a spese dello Stato, con tutele rafforzate per le vittime di tratta $\left(\right.$ art. 17) ${ }^{38}$.

In attuazione delle disposizioni della legge n. 47/2017 il Governo ha trasmesso alle Camere, per il prescritto parere, uno schema di regolamento che modifica quello attuativo del t.u. sull'immigrazione in vigore ${ }^{39}$.

Per il raggiungimento delle finalità di prevenzione generale dei reati ai danni dei minori stranieri non accompagnati, realizzata attraverso modalità significative di inserimento protetto nella società, le misure delle quali si propone l'introduzione nel regolamento sull'immigrazione, hanno portata decisiva. Incidono infatti sul rilascio dei permessi di soggiorno e sulla loro conversione in ordinario permesso di soggiorno al raggiungimento della maggiore età. A questo proposito il regolamento sull' immigrazione, quindi, recepirà la semplificazione delle tipologie di permesso di soggiorno per i minori stranieri non accompagnati, disciplinando il permesso per minore età o per motivi familiari dei quali si è detto, regolando in termini attuativi la possibilità di svolgere attività lavorativa e formativa finalizzata all' accesso al lavoro, nel rispetto delle norme sul lavoro minorile, prevedendo in maniera specifica la conversione del titolo in vero e proprio permesso di soggiorno al compimento della maggiore età, anche nel caso di diniego della protezione internazionale.

\section{Bibliography}

Antonini E., La tutela penale dei minori nel Testo Unico sull'immigrazione, [in:] Diritti umani degli immigrati. Tutela della famiglia e dei minori, ed. R. Pisillo Mazzeschi, P. Pustorno, A. Viviani, Napoli 2010.

Anzaldi A., Guarnier T. (ed.), Viaggio nel mondo degli stranieri non accompagnati: un' analisi giuridico-fattuale, Vol. II, In bilico fra il diritto al lavoro e lo sfruttamento, http://www.fondazionebasso.it.

Bertozzi R., Le politiche sociali per i minori stranieri non accompagnati, Milano 2005.

Bianchelli C., Il (dis)crimine della tratta. Un'indagine etnografica dei processi penali per riduzione in schiavitù e tratta di esseri umani, "Problemi del socialismo" 2016, LV.

38 Va rilevato che l'art. 16 della 1. n. 17 luglio 2020, n. 77 che converte il Dl 19 maggio 2020, n. 34, il c.d. "Decreto rilancio» in materia di Covid-19, dispone che i posti disponibili nelle strutture del Sistema di protezione, per un termine non superiore ai sei mesi successivi alla cessazione dello stato di emergenza, possano essere utilizzati per l'accoglienza dei richiedenti protezione internazionale. Questo comporta la possibilità, secondo la prima e controversa interpretazione, che tutti i minori stranieri non accompagnati possano accedere anche ai servizi dei quali godono i titolari di protezione internazionale.

39 Trattasi della proposta di Regolamento recante modifiche al D.P.R. 31 agosto 1999, n. 394, in attuazione dell' articolo 22 della legge 7 aprile 2017, n. 47, recante misure di protezione dei minori stranieri non accompagnati. Si veda: Atti del governo sottoposti a parere, n. 181, www.camera.it. 
Bichi R. (ed.), Separated children. I minori stranieri non accompagnati, Milano 2008.

Borello R., Il diritto all 'unità familiare nel diritto dell'immigrazione: riflessioni generali di diritto costituzionale interno e comparato, [in:] Diritti umani degli immigrati. Tutela della famiglia e dei minori, ed. R. Pisillo Mazzeschi, P. Pustorno, A.Viviani, Napoli 2010.

Cannavale A. G., Lazzari C., Tratta di persone (voce), [in:] Dig. Disc. Pen. terzo aggiornamento, Torino 2005.

Cianciolo V., La tutela del minore straniero non accompagnato, Maggioli 2019.

Di Nuzzo A., Minori migranti. Nuove identità transculturali, Carocci 2020.

Gambini M., I diritti del minore vittima di tratta e gli strumenti di tutela della persona, “Costituzionalismo.it” 2014.

Il sistema normativo a tutela dei minori stranieri non accompagnati, Unhcr, Unicef e altri, www.unhcr.it;

La tutela dei minori stranieri non accompagnati. Manuale giuridico per l'operatore, Asgi, www.asgi.it

La tutela dei minori stranieri non accompagnati. Manuale operativo, Ministero dell'Interno - Anci, www.siproimi.it

Lamarque E., Prima $i$ bambini. Il principio dei best interests of the child nella prospettiva costituzionale, Milano 2016.

Manuale sul diritto europeo in materia di asilo, frontiere e immigrazione, Agenzia dell' Unione europea per i diritti fondamentali 2014, https://fra.europa.eu

Marzetti J., Tutori volontari per minori stranieri non accompagnati, Rimini 2019.

Melossi D., Giovannetti M., I nuovi sciuscià: minori stranieri in Italia, Roma 2002.

Miazzi L., Il rimpatrio assistito del minore straniero: ancora un caso di diritto speciale?, "Diritto Immigrazione e Cittadinanza" 2000, no 2.

Minori stranieri non accompagnati, Camera dei deputati, XVIII legislatura, https://temi. camera.it/leg18/temi/tl18_minori_stranieri_non_accompagnati.html

Nicodemi F., Le vittime della tratta di persone nel contesto della procedura di riconoscimento della protezione internazionale: quali misure per un efficace coordinamento tra $i$ sistemi di protezione e di assistenza? "Diritto, immigrazione e cittadinanza” 2017, no 1.

Norme sull'accertamento dell'età dei minori stranieri non accompagnati, ASGI (23.01.2017), www.asgi.it

Piccoli schiavi invisibili 2019. Rapporto sui minori vittime di tratta e grave sfruttamento, Save the Children, IX edizione, https://s3.savethechildren.it

Piccoli schiavi invisibili 2020. L'impatto del COVID-19 sulla tratta e lo sfruttamento: dalle strade all'online, Save the Children, https://s3.savethechildren.it

Picotti L., La tutela penale del minore vittima di reato: profili sistematici e spunti critici, 2018, http://www.aiafrivista.it

Proposta di Regolamento recante modifiche al Dpr 31 agosto 1999, n. 394, in attuazione dell' articolo 22 della legge 7 aprile 2017, n. 47, recante misure di protezione dei minori stranieri non accompagnati, [in:] Atti del governo sottoposti a parere, Camera dei deputati, www.camera.it 
Report mensile minori stranieri non accompagnati (msna) in Italia, Dati al 31 dicembre 2019, Ministero del lavoro e delle politiche sociali, Direzione Generale dell'Immigrazione e delle Politiche di Integrazione, www.lavoro.gov.it

Summerer K., I delitti di schiavitù e tratta delle persone, [in:] Trattato di diritto penale, Vol. VIII, ed. A. Cadoppi, S. Canestrari, A. Manna, M. Papa, , Torino 2010.

Tarzia G., Il minore straniero non accompagnato: quale tutela nel sistema legislativo italiano?, "Minori Giustizia" 2008, no 3.

Triestina B., I minori stranieri non accompagnati. Analisi ragionata della L. 7 aprile 2017, n. 47, Piacenza 2017.

\section{SUMMARY}

\section{The criminal protection of the migrant minor in Italy}

Within the framework of the broader legal aim of the criminal safeguard of the minor, the protection addressed to him/her as a migrant is a specification that standardizes injurious modalities, of damage or endangerment, which mature in specifically harmful situations or conditions. In this regard, "dedicated" rules are necessary when the general ones, albeit accompanied by other forms of criminal and extra-criminal protection, cannot adequately achieve the purpose, as urged by important international commitments undertaken by Italy, aimed at facing the tragic data experience of the large number of situations in which no form of direct protection is feasible. In these cases, it is necessary through criminal laws, to build adequate shields to intervene with forms of protection that break the ties between minors and the criminal organizations to which the trafficking of human beings is linked. In some cases, criminal protection is the only remedy to safeguard those who, combining the conditions of a minor and a migrant, are doubly weak and therefore so vulnerable as to make the two general protections - as a minor and as a migrant - inadequate if they are called, alone, to face this extremely serious problem, without combining them with each other in the overall protection of migrant minors. The protection thus becomes "double" and "strengthened" and reaches, in a holistic way, a greater effectiveness than the sum of the two single manifestations, in pursuing the injury or endangerment of a series of primary legal assets (life, personal safety, freedom, self-determination, right to education and protection, etc.) which, to the detriment of these recipients, often take on extreme and hateful connotations or determine irreversible prejudices in growth and future life.

The criminal law in these cases specializes or aggravates some crimes of which the migrant can become a victim at every stage of life but which, when a minor, are in any case particularly serious or which, when the victim is a minor, configure more relevant risk profiles and of negative value. In cases where, on the other hand, the particular detrimental value of the fact committed against the minor does not involve the need to remodel the typical crime, it will take on importance in quantifying the 
sentence, because the crime will be more severe pursuant to art. 133 c.p. as for of the mode of action. To make greater protection effective, the system must safeguard it; first of all, in terms of public safety, through the effective contrast of the dark number and impunity, strengthening the ability to eliminate pitfalls and critical situations to the detriment of migrant minors, first of all those concerning unaccompanied foreign minors, which a recent law specifically deals with, implementing a strong international action. In fact, it is necessary that the social and police policies of the individual states should not bear the full weight of prevention which, however, when fragmented, cannot affect the specific criminal aspects of the entire phenomenon. National laws, especially in emergencies and in the presence of public opinion frightened by security clichés, often escape the transnational dimension of the problems out of mere opportunism, in order not to take charge of them in part-share. This is clearly demonstrated by the difficulties that have arisen to accommodate proportionately refugees and to globally oppose transnational crime, within which the most detrimental and most unpunished situations ripen to the damage of our specific legal interest in this field. 